\title{
Fatores associados ao descontrole glicêmico de diabetes mellitus em pacientes atendidos no Sistema Único de Saúde no Sudoeste da Bahia
}

\author{
Associated factors with uncontrolled glycemic status in \\ diabetic patients treated in the Unified Health System in \\ Southwest of Bahia
}

\author{
Claudio Lima Souza' (1), Marcio Vasconcelos Oliveira' \\ 'Instituto Multidisciplinar em Saúde, Universidade Federal da Bahia, Campus Anísio Teixeira - Vitória Conquista (BA), Brasil.
}

Como citar: Souza CL, Oliveira MV. Fatores associados ao descontrole glicêmico de diabetes mellitus em pacientes atendidos no Sistema Único de Saúde no Sudoeste da Bahia. Cad Saúde Colet, 2020;28(1):153-164. https://doi.org/10.1590/1414-462X202028010319

\begin{abstract}
Resumo
Introdução: $O$ diabetes mellitus constitui importante problema de saúde pública, responsável por grande morbidade e ônus significativo à saúde pública. A hemoglobina glicada, que avalia a glicemia média nos últimos sessenta dias, tem sido importante ferramenta na prevenção das comorbidades. Objetivo: Avaliar a prevalência do descontrole glicêmico e metabólico e fatores associados em indivíduos atendidos em um laboratório central do sudoeste da Bahia Método: Trata-se de estudo transversal realizado com 352 pacientes diabéticos atendidos em um laboratório do Sistema Único de Saúde. Resultados: O estudo demonstrou que mais de dois terços dos pacientes (70,2\%) apresentaram descontrole glicêmico. Análise multivariada apontou que receber menos visitas dos agentes comunitários OR 1,96 $(1,05$ - 3,65) e não possuir comorbidades associadas à doença OR 1,86 (1,05 - 3,30) estiveram associados a um pior controle glicêmico. Conclusão: Os resultados sugerem necessidade da melhoria na atenção dos pacientes com uma equipe de saúde sensível às práticas adequadas para melhor controle metabólico. Uma melhor ação da equipe de saúde pode contribuir para a promoção da saúde e melhor qualidade de vida destes pacientes e impactar em redução de custos com saúde por diminuição de internações e procedimentos relacionados às complicações do diabetes.
\end{abstract}

Palavras-chave: diabetes mellitus; hemoglobina glicada; controle.

\begin{abstract}
Background: Diabetes mellitus is an important public health issue, responsible for great morbidity and significant public health burden. A glycated hemoglobin, which assessed mean blood glucose in the past sixty days, has been an important tool in preventing comorbidities. Objective: To evaluate the prevalence of glycemic and metabolic uncontrolled and associated factors used in the central laboratory of southwest Bahia Method:This is a cross-sectional study conducted with 352 diabetic patients treated at the Laboratory of the Unified Health System. Results: The study demonstrated more than two thirds of the patients (70.2\%) described lack of glycemic control. Multivariate analysis showed that receiving fewer visits from community health workers OR 1.96 (1.05 - 3.65) and not have comorbidities associated OR 1.86 (1.05 - 3.30) were associated with poor glycemic control. Conclusion: The results suggested the need to improve patient care with a health team sensitive to the practices for better metabolic control. The best action of the health team can contribute to health promotion and better quality of life for patients and impact on the reduction of health costs by reducing hospitalizations and procedures related to diabetes complications.
\end{abstract}

Keywords: diabetes mellitus; glycated hemoglobin; control.

Este é um artigo publicado em acesso aberto (Open Access) sob a licença Creative Commons Attribution, que permite uso, distribuição e reprodução em qualquer meio, sem restrições desde que o trabalho original seja corretamente citado.
Trabalho realizado no Laboratório Central - Vitória da Conquista (BA), Brasil.

Correspondência: Cláudio Lima Souza.E-mail: caulimas@gmail.com

Fonte de financiamento: nenhuma.

Conflito de interesses: nada a declarar.

Recebido em: Jul. 15, 2018. Aprovado em: Jun. 04, 2019 


\section{INTRODUÇÃO}

O Diabetes Mellitus (DM) é uma síndrome de etiologia múltipla caracterizada como um grupo de desordens metabólicas marcado por hiperglicemia, resultante de falhas na secreção de insulina ou em sua função, além de defeitos genéticos das células beta ou processos patológicos que danificam o pâncreas. Está classificado em DM tipo 1, tipo 2, outros tipos e o diabetes gestacional ${ }^{1,2}$. O diabetes tipo 2 constitui uma das dez principais causas de morte no mundo ${ }^{3}$.

O DM é considerado a quinta maior causa de morte no mundo, com projeções para 2025 de 300 milhões de diabéticos, correspondente a 5,4\% da população mundial ${ }^{4,5}$. No Brasil, estima-se um número de 12 milhões de diabéticos e prevalência em torno de $8 \%$ na população de 30 a 69 anos. Todavia, este problema pode ser maior, pois metade dos indivíduos doentes pode desconhecer a sua condição, além de projetar-se futuramente uma maior mortalidade proporcional por diabetes mellitus ${ }^{6,7}$.

As complicações crônicas relacionadas ao DM são geralmente classificadas como microvasculares (retinopatia, nefropatia e neuropatia) e macrovasculares (doença arterial coronariana, doença cerebrovascular e vascular periférica) que são responsáveis por expressiva morbimortalidade e significativas taxas de mortalidade cardiovascular e renal, cegueira, além de perdas de função e amputação de membros ${ }^{8}$.

Complicações relacionadas ao estado hiperglicêmico, como falência renal e retinopatia e neuropatia diabéticas, são apontadas como responsáveis pela necessidade de oferta de elevado número de leitos hospitalares e cuidados médicos de elevado custo9.

A estratégia de prevenção das complicações crônicas do diabetes baseia-se no controle da hiperglicemia para tratamento precoce de suas complicações. É consenso a necessidade da manutenção de um controle glicêmico satisfatório em todos os pacientes, isto é, um grau de controle que previna a sintomatologia aguda e crônica atribuída à hiperglicemia e à hipoglicemia ${ }^{10}$.

Entre os parâmetros laboratoriais utilizados para acompanhar os pacientes estão glicemia de jejum, frutosamina e hemoglobina glicada ( $\mathrm{HbA1C}$ ). Esta constitui um importante preditor de complicações da doença, refletindo os níveis glicêmicos dos últimos três meses e tem sido utilizada como indispensável ferramenta de avaliação do controle glicêmico destes pacientes ${ }^{11}$.

Importantes estudos clínicos como Diabetes Control and Complications Trial (DCCT), em 1993, e do United Kingdom Prospective Diabetes Study (UKPDS), em 1998, revelaram que a manutenção do nível de $\mathrm{A} 1 \mathrm{C}$ abaixo de $7 \%$, no paciente diabético, proporcionaria redução considerável no risco para o desenvolvimento de complicações crônicas do DM ${ }^{12}$.

A nefropatia está presente em $15 \%$ a $20 \%$ dos pacientes com DM2 e em $30 \%$ a $40 \%$ dos diabéticos tipo 1 com longa evolução. Trata-se da principal causa de insuficiência renal em pacientes que fazem diálise. Os dados combinados do DCCT demonstram que o controle glicêmico satisfatório do DM1 pode reduzir a incidência de microalbuminúria em 39\% e de albuminúria em $54 \%$. A retinopatia diabética acomete cerca de $40 \%$ dos pacientes diabéticos e é a principal causa de cegueira em pacientes entre 25 e 74 anos. O DCCT mostrou que o controle satisfatório da glicemia pode reduzir a incidência de retinopatia em $76 \%$ e sua taxa de progressão em $54 \%^{13}$.

Os pacientes diabéticos têm em torno de 15 a 40 vezes mais risco de sofrer amputações do que os não diabéticos, e aproximadamente $20 \%$ dos amputados morrem em 2 anos ${ }^{14}$.

Para controle mais efetivo da doença e prevenção das complicações, a SBD (baseado nas recomendações da American Diabetes Asssociation) recomenda as seguintes metas: $\mathrm{HbA} 1 \mathrm{C} \mathrm{em}$ torno de $7 \%$ nos adultos e tolerável até $8,5 \%$ em idosos; PA inferior a $130 \times 80 \mathrm{mmHg}$; colesterol total inferior a $200 \mathrm{mg} / \mathrm{dl}$ e triglicérides menor que $150 \mathrm{mg} / \mathrm{dl}^{15}$.

O controle intensivo desses fatores através de medidas não farmacológicas e farmacológicas pode reduzir quase todas as complicações em pelo menos metade. Parte expressiva do acompanhamento do indivíduo com diabetes deve ser dedicada à prevenção, identificação e manejo destas complicações. O manejo requer uma equipe de atenção básica treinada com tarefas específicas, incluindo a coordenação do plano terapêutico e das referências e contra referências dentro do sistema de saúde ${ }^{8}$. 
O objetivo deste trabalho foi avaliar fatores associados ao descontrole glicêmico em pacientes atendidos para realização de exames laboratoriais no Sistema Único de Saúde em Vitória da Conquista - BA, através de entrevista semiestruturada e coleta de dados de prontuário.

\section{MÉTODO}

Trata-se de um estudo transversal baseado em entrevista semiestruturada e coleta de dados em prontuário realizados com pacientes do sudoeste da Bahia no período de julho de 2014 a fevereiro de 2015.

\section{População}

No ano de 2014, a estimativa de população para o município compreendia 340.199 indivíduos. A cobertura da atenção primária no mesmo ano era de $51 \%$ e pessoas de idade superior a 20 anos realizavam exames no Laboratório Central de Saúde Pública. A fração populacional correspondente a esta faixa etária engloba 227.933 pessoas e 116.246 representam a população maior de 20 anos coberta pela Estratégia de Saúde a Família no município ${ }^{16,17}$. A partir deste número foi efetuada amostragem aleatória simples, resultando numa estimativa amostral de 382 indivíduos, com precisão de $5 \%$ e intervalo de $95 \%$. Devido à heterogeneidade dos desfechos estudados considerou-se uma prevalência de $50 \%$.

Foram considerados critérios de inclusão neste estudo, portadores de diabetes mellitus com idade superior a 20 anos (diagnosticados por exame laboratorial ou pelo relato de uso de antidiabéticos orais ou insulina), que aceitaram participar da pesquisa e assinaram o termo de consentimento livre e esclarecido.

Este estudo foi aprovado pelo Comitê de ética da Universidade Federal da Bahia - IMS/CAT, protocolo no 20208513.8.0000.5556.

Os dados foram obtidos por meio de entrevista no laboratório utilizando-se questionário semiestruturado adaptado da Pesquisa Nacional de Saúde ${ }^{18}$. Os dados foram coletados e armazenados em netbooks utilizando o programa EPI INFO, versão 3.5.1.

\section{Evento e variáveis explicativas}

A variável dependente deste estudo foi o descontrole glicêmico do DM, representado pela hemoglobina glicada superior a 7\% ou glicemia de jejum superior a 130 mg/dl na ausência de HbA1C.

Variáveis explicativas potenciais avaliadas neste estudo compreenderam: 1) sociodemográficas (sexo, idade, estado conjugal, renda, escolaridade, autoavaliação do estado de saúde, prática de atividade física e etilismo); 2) clínicas (diagnóstico de hipertensão arterial, doenças crônicas associadas, medida de pressão arterial); e 3) relacionadas ao serviço (consulta médica, cadastramento na UBSF, número de visitas do ACS e índice de acesso aos serviços de saúde).

Para aferição das medidas antropométricas foram utilizados: balança eletrônica portátil Marte, modelo LC200 $\mathrm{pp}^{\mathrm{TM}}$, com capacidade de $200 \mathrm{~kg}$, para medida do peso; Estadiômetro portátil CAUMAQ ${ }^{T M}$, modelo Est-22A, para aferição da altura; e esfigmomanômetro digital Omron HEM-742 ${ }^{\mathrm{TM}}$, para aferição pressão arterial. Todas as medidas seguiram recomendações de padronização ${ }^{19}$. Os exames laboratoriais foram coletados no Laboratório Central, processados através do Sistema Complab 3.0 e os resultados, tabulados em planilhas do software Microsoft Office Excel ${ }^{\mathrm{TM}}$.

A autoavaliação da saúde foi agrupada em três categorias: boa/muito boa, regular, ruim/muito ruim. E a prática de atividade física foi definida como resposta positiva à pergunta "Nos últimos três meses, o(a) sr(a) praticou algum tipo de exercício físico ou esporte (caminhada, baba, capoeira, etc.)?" 


\section{Análise estatística}

A análise estatística partiu de uma análise descritiva seguida das variáveis categóricas através de frequência relativa e das variáveis contínuas por medidas de tendência central através de média e mediana. A estimativa de pacientes em descontrole da doença foi estimada pela proporção de indivíduos sabidamente diabéticos com níveis de HBA1C superiores a 7\%, glicemia de jejum superior a $130 \mathrm{mg} / \mathrm{dl}$ ou não ter realizado exames de glicemia ou HBA1C nos últimos três meses ${ }^{20}$. Foi considerada significância estatística $5 \%$ para a análise univariada usando teste qui-quadrado e a associação entre a variável resposta e as explicativas foi estimada pela Odds Ratio, considerando intervalo de $95 \%$ de confiança. Para avaliação dos fatores independentemente relacionados ao desfecho, foi realizada análise multivariada por meio de regressão logística. O modelo inicial incluiu variáveis com valor de $\mathrm{p}<0,20$ na análise univariada e valor $\mathrm{p}<0,05$ definiu a significância estatística para permanência no modelo final. $\mathrm{O}$ ajustamento do modelo foi avaliado pelos critérios de Akaike. As análises foram realizadas utilizando os pacotes estatísticos $R$ versão 2.11.1 e EPI INFO versão 7.0.9.7.

\section{RESULTADOS}

Fizeram parte desta análise, 352 indivíduos devido à perda amostral ocasionada por falta de dados no sistema. Foram considerados em descontrole glicêmico consoante critérios estabelecidos, 70,2\% (247) dos indivíduos estudados. O valor médio de HBA1C na população foi de $8,7 \%$ (DP 2,4\%) e a última glicemia de jejum revelou uma média de 169,8 mg/dl (DP 74mg/dl).

A população analisada foi composta por adultos maiores de 20 anos. A maior parte da amostra foi representada por mulheres $(69,6 \%)$ e $71,6 \%$ dos entrevistados possuíam renda igual ou inferior a um salário mínimo (R\$ 722,00, à época do estudo). Além disso, $53,1 \%$ dos respondentes relataram ter nenhuma escolaridade ou elementar incompleta e $47,7 \%$ disseram não ter companheiro (Tabela 1).

Tabela 1. Análise descritiva da população estudada para controle glicêmico e fatores associados em diabéticos na cidade de Vitória da Conquista - BA, 2015

\begin{tabular}{|c|c|c|}
\hline Variável & $\mathbf{N}$ totala $^{a}$ & $\%$ \\
\hline \multicolumn{3}{|l|}{ Faixa etária } \\
\hline $22-59$ anos & 162 & 46,5 \\
\hline$\geq 60$ anos & 186 & 53,5 \\
\hline \multicolumn{3}{|l|}{ Sexo } \\
\hline Feminino & 243 & 69,6 \\
\hline Masculino & 106 & 30,4 \\
\hline \multicolumn{3}{|l|}{ Escolaridade } \\
\hline Sem instrução/elementar incompleto & 185 & 53,0 \\
\hline Elementar completo ou mais & 164 & 47,0 \\
\hline \multicolumn{3}{|l|}{ Renda } \\
\hline Maior que um salário mínimo b & 100 & 28,4 \\
\hline Até um salário minimo ${ }^{\text {b }}$ & 252 & 71,6 \\
\hline \multicolumn{3}{|l|}{ Estado conjugal } \\
\hline Sem companheiro & 168 & 47,7 \\
\hline Com companheiro & 184 & 52,3 \\
\hline
\end{tabular}


Tabela 1. Continuação...

\begin{tabular}{lcc}
\hline \multicolumn{1}{c}{ Variável } & N total & \\
\hline Trabalha atualmente & 102 & 29,2 \\
\hline Não & 247 & 70,8 \\
\hline Sim & & 20,2 \\
\hline Autoavaliação do estado de saúde & 70 & 50,6 \\
\hline Boa/Muito boa & 175 & 29,2 \\
\hline Regular & 101 & 91,3 \\
\hline Ruim/Muito ruim & & 8,7 \\
\hline Atualmente fuma ou já fumou? & 314 & \\
\hline Não & 30 & 79,6 \\
\hline Sim & & 20,4 \\
\hline Depressão (Score PHQ9) & 280 & \\
\hline Não & 72 & \\
\hline Sim & 105 & \\
\hline Controle glicêmico ** & 247 & \\
\hline Não & & \\
\hline Sim & 105 & \\
\hline
\end{tabular}

Diagnóstico médico de hipertensão arterial

\begin{tabular}{lc}
\hline Não & 96 \\
\hline Sim & 252 \\
\hline
\end{tabular}

Histórico familiar de diabetes na família

\begin{tabular}{ll}
\hline Não & 47 \\
\hline Sim & 298 \\
\hline
\end{tabular}

Visita ao médico regularmente por causa do diabetes

\begin{tabular}{lc}
\hline Não & 38 \\
\hline Apenas quando tem problema & 44 \\
\hline Sim & 260 \\
\hline Circunferência de Cintura & 125 \\
\hline Normal & 227 \\
\hline Aumentada & \\
\hline Visita do agente comunitário de saúde & 207 \\
\hline Mensal ou bimestral & 60 \\
\hline Três a quatro vezes ao ano ou menos
\end{tabular}

Prática autorreferida de exercício ou esporte nos últimos três meses

\begin{tabular}{ll} 
Não & 148 \\
\hline Sim & 233 \\
\hline
\end{tabular}

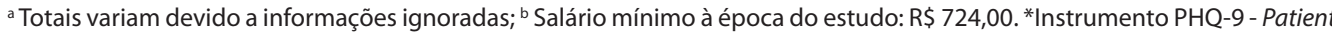
Health Questionnaire; **HbA1C $>7 \%$ ou Glicose jejum $>130$ 
Em relação à autopercepção de saúde, $79,8 \%$ das pessoas disseram ter saúde regular, ruim ou muito ruim. A prática de exercício físico nos últimos três meses foi admitida por $32,8 \%$ das pessoas.

Em relação às questões relacionadas ao $\mathrm{DM}, 86,3 \%$ disseram ter histórico familiar de diabetes, $73 \%$ dos indivíduos se declararam hipertensos, $10 \%$ precisaram de internamento no último ano e $75 \%$ visitavam regularmente o médico para tratar a doença.

Dentre os parâmetros de controle do diabetes para diminuição da ocorrência de comorbidades associadas ao descontrole glicêmico, 169 pacientes $(48,0 \%)$ possuíam pressão arterial alterada em aferição por triplicata no momento da entrevista, 208 (54,5\%) possuíam a última glicemia medida alterada (superior a 130 mg/dl) e 227 (64,5\%), circunferência de cintura aumentada corroborando com o IMC (Índice de Massa Corporal), que estava aumentado em $70,2 \%$ dos pacientes. A maioria dos indivíduos apresentou níveis de triglicerídeos normais, correspondendo a $63,1 \%$, e colesterol total normal em $72,4 \%$ (Tabela 2 ).

Tabela 2. Distribuição dos parâmetros de controle nos diabéticos para diminuição de complicações e/ou comorbidades na cidade de Vitória da Conquista - BA, 2015

\begin{tabular}{lcccc} 
Parâmetro & Média em mmHg & $\begin{array}{c}\text { Meta } \\
\text { desejada }\end{array}$ & $\begin{array}{c}\text { Pacientes em } \\
\text { controle Na (\%) }\end{array}$ & $\begin{array}{c}\text { Pacientes em } \\
\text { Descontrole } \\
\mathbf{N a}^{\mathbf{a}}(\%)\end{array}$ \\
\hline Pressão arterial $^{\mathbf{b}}$ & $138 / 82$ & $130 / 80$ & $183(52,0 \%)$ & $169(48,0 \%)$ \\
\hline HBA1C & $8,7 \%$ & $<7,0 \%$ & $81(30,3 \%)$ & $186(69,7 \%)$ \\
\hline Última glicemia & $170 \mathrm{mg} / \mathrm{dl}$ & $<130 \mathrm{mg} / \mathrm{dl}$ & $127(37,9 \%)$ & $208(62,1 \%)$ \\
\hline Colesterol total & $187 \mathrm{mg} / \mathrm{dl}$ & $<200 \mathrm{mg} / \mathrm{dl}$ & $266(75,6 \%)$ & $86(24,4 \%)$ \\
\hline Colesterol LDL & $104 \mathrm{mg} / \mathrm{dl}$ & $<130 \mathrm{mg} / \mathrm{dl}$ & $225(81,8 \%)$ & $50(18,2 \%)$ \\
\hline Colesterol HDL & $49 \mathrm{mg} / \mathrm{dl}$ & $>49 \mathrm{mg} / \mathrm{dl}$ & $67(35,6 \%)$ & $121(64,4 \%)$ \\
\hline Triglicerídeos & $194 \mathrm{mg} / \mathrm{dl}$ & $<150 \mathrm{mg} / \mathrm{dl}$ & $133(49,4 \%)$ & $136(50,6 \%)$ \\
\hline IMC & 29 & Até 25 & $97(27,6 \%)$ & $255(72,4 \%)$ \\
\hline
\end{tabular}

a Número e proporção de pacientes para cada categoria; ${ }^{\mathrm{a}}$ Pressão arterial aferida em campo por esfigmomanômetro digital

A análise univariada indicou que as variáveis "estado conjugal sem companheiro"," praticar exercício físico nos últimos três meses" $\mathrm{e}$ "possuir morbidades associadas ao diabetes" estiveram estatisticamente associadas ao descontrole glicêmico $(p<0,05)$. A maior periodicidade da visita domiciliar do agente comunitário de saúde aos pacientes esteve também associada a maior descontrole, com uma OR 1,63 (IC - 0,92-2,87) com significância estatística de p=0,06 (Tabela 3).

Tabela 3. Análise univariada da população estudada para controle glicêmico e fatores associados em diabéticos na cidade de Vitória da Conquista - BA, 2015

\begin{tabular}{|c|c|c|c|c|}
\hline Variável & $\mathbf{N}$ & DES (\%) ${ }^{a}$ & OR (IC 95\%) & Valor de $p^{c}$ \\
\hline \multicolumn{5}{|l|}{ Faixa etária } \\
\hline$\geq 59$ anos & 186 & $133(71,5)$ & 1 & \\
\hline $20-59$ anos & 162 & $111(68,5)$ & $0,87(0,55-1,37)$ & 0,31 \\
\hline \multicolumn{5}{|l|}{ Sexo } \\
\hline Feminino & 243 & $174(71,6)$ & 1 & \\
\hline Masculino & 106 & $72(67,9)$ & $1,19(0,73-1,95)$ & 0,28 \\
\hline
\end{tabular}

a Totais variam devido a informações ignoradas; *Instrumento PHQ-9 - Patient Health Questionnaire 
Tabela 3. Continuação...

\begin{tabular}{|c|c|c|c|c|}
\hline Variável & $\mathbf{N}$ & DES (\%) ${ }^{\text {a }}$ & OR (IC 95\%) & Valor de $p^{c}$ \\
\hline \multicolumn{5}{|l|}{ Escolaridade } \\
\hline Elementar completo ou mais & 185 & $129(69,7)$ & 1 & \\
\hline Sem instrução/elementar incompleto & 164 & $116(70,7)$ & $0,95(0,60-1,51)$ & 0,47 \\
\hline \multicolumn{5}{|l|}{ Estado conjugal } \\
\hline Com companheiro & 168 & $110(65,5)$ & 1 & \\
\hline Sem companheiro & 184 & $137(74,6)$ & $1,54(0,97-2,43)$ & $<0,05$ \\
\hline \multicolumn{5}{|l|}{ Trabalha atualmente } \\
\hline Sim & 247 & $174(70,5)$ & 1 & \\
\hline Não & 102 & $71(69,6)$ & $0,96(0,58-1,59)$ & 0,49 \\
\hline \multicolumn{5}{|l|}{ Autoavaliação do estado de saúde } \\
\hline Regular/Ruim/Muito ruim & 276 & $195(70,7)$ & 1 & \\
\hline Boa/Muito boa & 70 & $48(68,6)$ & $0,91(0,51-1,6)$ & 0,42 \\
\hline \multicolumn{5}{|l|}{ Visita do agente comunitário de saúde } \\
\hline Mensal ou bimestral & 220 & $126(57,3)$ & 1 & \\
\hline Três a quatro vezes ao ano ou menos & 62 & $28(45,2)$ & $1,63(0,92-2,87)$ & 0,06 \\
\hline \multicolumn{5}{|c|}{ Visita ao médico regularmente por causa do diabetes } \\
\hline Sim & 260 & $179(68,9)$ & 1 & \\
\hline Não & 38 & $27(71,0)$ & $1,11(0,52-2,35)$ & 0,48 \\
\hline Apenas quando tem problema & 44 & $35(79,6)$ & $1,76(0,81-3,83)$ & 0,10 \\
\hline \multicolumn{5}{|l|}{ Depressão } \\
\hline Sim & 72 & $47(65,3)$ & 1 & \\
\hline Não & 280 & $200(71,4)$ & $1,33(0,76-2,31)$ & 0,19 \\
\hline \multicolumn{5}{|l|}{ Circunferência de Cintura } \\
\hline Aumentada & 227 & $161(70,9)$ & 1 & \\
\hline Normal & 125 & $86(68,6)$ & $0,90(0,56-1,45)$ & 0,38 \\
\hline \multicolumn{5}{|c|}{ Prática de exercício físico nos últimos três meses autorreferida } \\
\hline Não & 233 & $155(66,5)$ & 1 & \\
\hline Sim & 119 & $92(77,3)$ & $1,71(1,03-2,85)$ & $<0,05^{*}$ \\
\hline \multicolumn{5}{|l|}{ Atualmente o senhor(a) fuma? } \\
\hline Não & 314 & $217(69,1)$ & 1 & \\
\hline Sim & 30 & $23(76,7)$ & $0,68(0,23-1,64)$ & 0,26 \\
\hline \multicolumn{5}{|l|}{ Depressão (Score PHQ-9)* } \\
\hline Sim & 72 & $47(65,3)$ & 1 & \\
\hline Não & 280 & $200(71,4)$ & $1,33(0,76-2,30)$ & 0,19 \\
\hline \multicolumn{5}{|c|}{ Morbidades ou complicações associadas ao diabetes } \\
\hline Sim & 114 & $74(64,9)$ & 1 & \\
\hline Não & 229 & $167(72,9)$ & $1,46(0,9-2,36)$ & 0,08 \\
\hline
\end{tabular}

a Totais variam devido a informações ignoradas; *Instrumento PHQ-9 - Patient Health Questionnaire 
Utilizando modelo de regressão logística, foram introduzidas variáveis com $p<0,20$ e permaneceram no modelo final ajustado apenas as variáveis com significância estatística $(p<0,05)$. O modelo logístico revelou que estiveram independentemente associadas ao descontrole glicêmico as seguintes variáveis: visita domiciliar do agente comunitário de saúde três a quatro vezes por ano ou menos ( $\mathrm{OR}=1,96, \mathrm{IC} 1,05-3,65)$, prática de exercício físico nos últimos três meses $(\mathrm{OR}=1,95, \mathrm{IC} 1,06-3,58)$ e possuir morbidades associadas ao diabetes $(\mathrm{OR}=1,86,1,05$ - 3,30) (Tabela 4). A variável "estado conjugal sem companheiro" permaneceu no modelo multivariado final por melhor ajustamento apontado pelos critérios de Akaike.

Tabela 4. Análise multivariada da população estudada para controle glicêmico e fatores associados em diabéticos na cidade de Vitória da Conquista - BA, 2015

\begin{tabular}{|c|c|c|}
\hline Variável & Odds Ratio (IC 95\%) & Valor de $\mathbf{p}^{\mathrm{a}}$ \\
\hline \multicolumn{3}{|c|}{ Visita domiciliar do agente comunitário de saúde } \\
\hline Mensal ou bimestral & 1 & \\
\hline Três a quatro vezes ao ano ou menos & $1,96(1,05-3,65)$ & $<0,05^{\mathrm{a}}$ \\
\hline \multicolumn{3}{|c|}{ Praticou exercício físico nos últimos três meses } \\
\hline Sim & 1 & \\
\hline Não & $1,95(1,06-3,58)$ & $<0,05^{\mathrm{a}}$ \\
\hline \multicolumn{3}{|c|}{ Presença de outras doenças ou morbidades associadas ao DM } \\
\hline Sim & 1 & \\
\hline Não & $1,86(1,05-3,30)$ & $<0,05^{\mathrm{a}}$ \\
\hline \multicolumn{3}{|l|}{ Casado(a) ou possui companheiro(a) } \\
\hline Sim & 1 & \\
\hline Não & $1,60(0,92-2,78)$ & 0,09 \\
\hline
\end{tabular}

a Valores significantes $(p<0,05)$

\section{DISCUSSÃO}

Foi detectada uma prevalência muito elevada $(70,2 \%)$ de descontrole glicêmico no grupo estudado e $72 \%$ dos pacientes entrevistados relataram nunca ter tido os pés examinados. $\mathrm{Na}$ perspectiva do controle glicêmico, foi considerada meta para HbA1c inferior a 7\%, pois está mais relacionada à redução primária e secundária do risco cardiovascular, conforme recomendações vigentes. Ressalta-se, no entanto, que a flexibilização e individualização da meta para cada paciente deve ser considerada, especialmente em pacientes idosos e com outras morbidades ${ }^{2}$. Cumpre ressaltar que, nesta análise, nem todos os pacientes realizaram $\mathrm{HbA} 1 \mathrm{C}$ e foram considerados em descontrole aqueles com glicemia maior que $130 \mathrm{mg} / \mathrm{dl}$ ou ainda não ter realizado exames laboratoriais nos últimos meses, conforme recomendação ${ }^{20}$. O simples fato de não ter realizado o exame HBA1C, que em níveis inferiores a $7 \%$ associam-se à redução acentuada da morbimortalidade, fragiliza o controle do diabetes e aumenta o risco de eventos mórbidos destes indivíduos.

Destaca-se ainda a necessidade de controle específico para os portadores de DM1, que, além da monitorização trimestral de $\mathrm{HbA} 1 \mathrm{C}$, requerem a verificação diária da glicemia capilar, que promove grandes benefícios e diminui risco de complicações agudas da doença, como cetoacidose ${ }^{21}$. Os objetivos glicêmicos diários são acompanhados através de glicosímetro e as metas de $\mathrm{HbA} 1 \mathrm{C}$ variam de acordo com situações clínicas, comorbidades associadas e faixas etárias. 
Os demais dados coletados também apontaram para descontrole da doença: $48 \%$ dos diabéticos estudados apresentaram descontrole da PA (PA superior a 130/80 $\mathrm{mmHg}$ ); mais da metade dos pacientes apresentaram níveis de glicose ou triglicérides ou colesterol HDL fora dos limites desejáveis e mais de dois terços dos diabéticos tinham IMC elevado. Estes dados de descontrole metabólico na maioria dos pacientes denotam preocupação devido à alta carga de morbidade associada às complicações ligadas ao DM não controlado. Estudos demonstram que o descontrole glicêmico está associado a mais complicações macro e microvasculares e impacta significantemente nos gastos com saúde ${ }^{22}$.

A maioria da população avaliada era do sexo feminino. Esta tendência já tem sido demonstrada em outros estudos em que as mulheres procuram mais pelo serviço de saúde e também aderem mais às práticas de saúde, especialmente quando se trata de adoção de medidas preventivas ${ }^{23}$.

Dados sociodemográficos apontaram ainda para uma população de baixa escolaridade e baixa renda, além de $47,7 \%$ relatarem não ter companheiro. A falta de companheiro apresentou associação com uma maior chance de descontrole glicêmico $(p=0,09)$ e permaneceu no modelo logístico final, estando associada ao descontrole glicêmico de forma independente. Alguns autores encontraram tal associação ${ }^{24}$, embora ainda seja controversa. Também não se pode afastar a causalidade reversa devido ao estudo ser de desenho transversal. A baixa escolaridade e renda não estiveram significativamente associadas com o descontrole da doença. Embora sabidamente escolaridade e renda estejam associadas à falta de cuidados em saúde, a homogeneidade da população atendida neste estudo (de baixa escolaridade e renda) não permitiu avaliar esta variável.

Em relação às variáveis de estado clínico, $72,6 \%$ dos diabéticos informaram diagnóstico prévio médico de hipertensão arterial coexistindo com DM. Dos diabéticos hipertensos deste estudo, $48 \%$ apresentaram PA aferida maior que $130 \times 90 \mathrm{mmHg}$. A associação entre DM e hipertensão é frequente e contribui para desenvolvimento de nefropatia diabética, principalmente quando a pressão não é adequadamente controlada ${ }^{15}$.

Outro dado que chama a atenção para incremento de risco cardiovascular é a circunferência de cintura aumentada em $64,5 \%$ dos indivíduos e IMC acima do desejável em $72,4 \%$. A associação de DM com aumento da gordura visceral também imputa maior risco para doença cardiovascular, especialmente quando associada à presença de hipertensão e $\mathrm{DM}^{25}$.

Quanto aos parâmetros laboratoriais de controle de dislipidemias, metade dos diabéticos $(50,6 \%)$ apresentou hipertrigliceridemia e $64,4 \%$ tinham HDL aquém do limite que confere proteção cardiovascular, denotando falta de controle nos parâmetros relacionados a dislipidemia. Intervenções farmacológicas adequadas também contribuem para a prevenção de DCV em $\mathrm{DM}^{26}$. Neste grupo, 31,1\% dos diabéticos relataram uso de vastatina. Este dado pode estar subnotificado devido a viés de memória, além de a população estudada ter baixa escolaridade. Ainda assim, o descontrole metabólico é patente na maioria dos parâmetros de controle estudados.

Não obstante o descontrole dos parâmetros metabólicos e clínicos relacionados ao DM, $76 \%$ dos pacientes relataram visita regular ao médico por causa da doença e $77,5 \%$ receberam visita mensal ou bimestral do agente comunitário de saúde. Aqueles que receberam visitas em maior intervalo de tempo apresentaram maior chance de descontrole glicêmico $1,63(0,92$ - 2,87), associação foi estatisticamente significativa na análise multivariada. Esta informação reafirma a importância dos ACS's no controle da doença, ainda que a maioria dos indivíduos não apresente controle adequado dos parâmetros propostos.

A prática de exercício físico traz benefícios e melhora o controle da doença, desde que seja bem orientada e esteja vinculada a um bom controle metabólico e nutricional. Neste estudo, a prática autorreferida de exercícios físicos ou esporte nos últimos meses esteve associada a um pior controle do estado glicêmico $O R=1,71(1,03-2,85), p<0,05$, ainda que a maioria $(61,1 \%)$ tivesse relatado não realizar tal prática. Estudos relacionados ao exercício e controle do DM são controversos no que diz respeito aos benefícios para controle da doença. No entanto, há compreensão de que interações entre uso de antidiabéticos orais, insulina gravidade da doença e comorbidades é essencial para otimização do controle glicêmico ${ }^{27}$. Embora a 
recomendação seja clara em razão dos benefícios nos estudos controlados com alto nível de evidência ${ }^{28}$, a prática de exercício deve ser orientada em razão das diferentes necessidades para os diferentes estados metabólicos dos pacientes e deve ser controlada em relação ao tempo, intensidade, presença de complicações e tempo de doença, além da terapia utilizada ${ }^{27,29-31}$. Ressalta-se que o município não dispunha de práticas de educação física supervisionada por profissional habilitado nas unidades de saúde da família e uma disponibilidade inadequada de nutricionistas, restrito apenas aos Núcleos de Assistência à Saúde da Família (NASF), contando com dois profissionais, à época do estudo. Além disso, cumpre ressaltar que as informações foram autorreferidas quanto à prática de exercício e não o tipo, intensidade e duração, o que também dificulta as inferências sobre sua efetividade, o que sinaliza para a necessidade de mais estudos que particularizem a prática de exercício com controle de variáveis e seu efeito sobre o controle glicêmico.

Destacou-se neste estudo a alta prevalência de pacientes diabéticos em descontrole glicêmico, falta de controle da pressão arterial, descontrole metabólico, além de notável falta de exames dos pés, embora a maioria tenha relatado acesso à unidade de saúde e realizado exame médico no último ano. Na análise multivariada, os fatores independentemente associados ao descontrole glicêmico apontaram variáveis associadas à vinculação do paciente ao serviço de saúde como preditoras de melhor controle glicêmico. Pacientes que receberam menos visitas do agente comunitário em saúde e sem outras doenças associadas diagnosticadas tiveram maior chance de descontrole glicêmico. Os dados apontam que receber menos cuidados à saúde relacionados ao $\mathrm{DM}$, seja pelo menor número de visitas do ACS ou pela menor presença ao serviço devido a não possuir comorbidades diagnosticadas associadas, está associado a um pior controle dos níveis glicêmicos neste grupo. Cumpre ressaltar ainda que os profissionais envolvidos no cuidado devem estar mais sensíveis às práticas de saúde no sentido de dirigir esforços para o controle metabólico dos indivíduos diabéticos, a fim de reduzir a morbidade associada e promover melhor qualidade de vida nesta população e, ainda, reduzir custos com a saúde pela diminuição de internações e procedimentos relacionados às complicações do diabetes.

O presente estudo apontou para a necessidade de melhorias na qualidade da atenção prestada aos pacientes portadores de diabetes na população estudada. Não obstante a maioria dos pacientes haverem tido acesso à consulta médica e aos medicamentos, foram verificadas importantes prevalências de descontrole glicêmico, metabólico e pressão arterial elevada. Chama a atenção ainda que mais de dois terços dos indivíduos não tiveram os pés examinados para prevenção do pé diabético, por se tratar de exame simples e de baixo custo.

Estiveram independentemente associados ao descontrole glicêmico um menor número de visitas do ACS às residências dos indivíduos, que associa-se diretamente ao cuidado; uma menor quantidade de comorbidades associadas ao DM, que impacta num menor número de visitas médicas e a prática de exercício físico, que, feito de forma inadequada, não produz os benefícios esperados e pode prejudicar o controle glicêmico.

Todos os fatores apresentados estão relacionados ao serviço de saúde e à qualidade da atenção prestada. É necessário que as práticas de saúde relacionadas ao controle do DM e prevenção de comorbidades sejam efetivamente postas em prática e a equipe de saúde esteja conscientizada e envolvida na necessidade de que os pacientes atinjam as metas de controle pré-estabelecidas pelas diretrizes das Sociedades médicas e orientações do Ministério da Saúde. Assim, poderá haver impacto na diminuição de comorbidades associadas, melhoria da qualidade de vida e menor impacto nos custos da saúde, como as complicações micro e macrovasculares do diabetes, que tanto oneram os cofres da saúde pública.

\section{REFERÊNCIAS}

1. Gross JL, Silveiro SP, Camargo JL, Reichelt AJ, Azevedo MJ. Diabetes melito: diagnóstico, classificação e avaliação do controle glicêmico. Arq Bras Endocrinol. 2002;46(1):16-26. http://dx.doi.org/10.1590/S000427302002000100004. 
2. Consenso Brasileiro sobre Diabetes. Diagnóstico e classificação do diabetes mellitus e tratamento do diabetes mellitus tipo 2 [Internet]. São Paulo: Sociedade Brasileira de Diabetes; 2000 [citado em 2013 maio 8]. Disponível em: http://bvsms.saude.gov.br/bvs/publicacoes/consenso_bras_diabetes.pdf

3. King H, Uubert RE, Herman WH. Global burden of Diabetes, 1995-2025: Prevalence, numerical estimates and projections [Internet]. Diabetes Care. 1998;21(9):1414-30 [citado em 2013 Fev 28]. Disponível em: http://www.inca.gov.br/requênci/docs/diabete.pdf

4. Aguiree F, Brown A, Cho NH, Dahlquist G, Dodd S, Dunning T, Scott C (2013). International Diabetes Dederation Diabetes Atlas. 6th ed. Brussels, Belgium: IDF.

5. Roglic G, Unwin N, Bennett PH, Mathers C, Tuomilehto J, Nag S, et al. The burden of mortality attributable to diabetes: realistic estimates for the year 2000. Diabetes Care. 2005;28(9):2130-5. http://dx.doi.org/10.2337/ diacare.28.9.2130. PMid:16123478.

6. Dias JCR, Campos JADB. Diabetes Mellitus: Reasons for prevalence in different geographic regions of Brazil, 2002 - 2007. Cien Saude Colet. 2012;17(1):239-44. http://dx.doi.org/10.1590/S1413-81232012000100026. PMid:22218557.

7. Lessa I. Tendência da mortalidade proporcional pelo diabetes mellitus nas capitais brasileiras, 1950-1985. Bol Oficina Sanit Panam. 1992;113(3):212-7. PMid:1418629.

8. Brasil. Ministério da Saúde. Secretaria de Políticas de Saúde. Departamento de Ações Programáticas Estratégicas. Diabetes Mellitus. Brasília: Ministério da Saúde, Departamento de Atenção Básica; 2006. (Caderno de Atenção Básica Série A. Normas e Manuais Técnicos, 16). p. 29-44.

9. Martins, AR. Hemoglobina glicada: ações para sua utilização com base no conhecimento científico. J Bras Patol Med Lab. 2003;39(3):198.

10. Duncan BB, Schmidt MI, Giugliani ERJ. Medicina ambulatorial: condutas clínicas em atenção primária. Porto Alegre: Artmed; 1996.

11. Lopes FM, Araújo ET, Silva KJ, Silva MC, Cruz RO, Lisboa ES. A avaliação da hemoglobina glicada como importante marcador do Diabetes mellitus. Ensaios e Ciência, C. Biológicas, Agrárias e da Saúde. 2011. 15(3):65-82.

12. Sumita NM. As interferências e as limitações metodológicas na dosagem da hemoglobina glicada (A1C). J Bras Patol Med Lab. 2012;48(5):312. http://dx.doi.org/10.1590/S1676-24442012000500001.

13. Nathan DM, Genuth S, Lachin J, Cleary P, Crofford O, Davis M, et al. Diabetes Control and Complications Trial (DCCT): The effect of intensive treatment of Diabetes on the development and progression of the long-term complications in insulin-dependent diabetes mellitus. N Engl J Med. 1993;329(14):977-86. http://dx.doi.org/10.1056/NEJM199309303291401. PMid:8366922.

14. Pitta, GBB, Castro AA, Soares AMMN, Maciel CJJ, Silva JDM, MunizVMT, et al. Perfil dos pacientes portadores de pé diabético atendidos no Hospital Escola José Carneiro e na Unidade de Emergência Armando Lages. J Vasc Br. 2005;4(1):5-10.

15. Santos AL, Cecílio HPM, Teston EF, Arruda GO, Peternella FMN, Marcon SS. Complicações microvasculares em diabéticos tipo fatores associados: inquérito telefônico de morbidade autorreferida. Cien Saude Colet. 2015;20:761770.

16. Instituto Brasileiro de Geografia e Estatística. Contagem Populacional [Internet]. 2013 [citado em 2013 fev 28]. Disponível em: http://www.ibge.gov.br/cidadesat/topwindow.htm?1

17. Vitória da Conquista. Secretaria Municipal de Saúde [Internet]. 2012 [citado em 2012 maio 23]. Disponível em: http://www.pmvc.ba.gov.br/v1/secretaria/163/saude.html

18. Pesquisa Nacional de Saúde. Inquérito Região Integrada do Distrito Federal (RIDE/DF) [Internet]. 2011 [citado em 2011 abr 2]. Disponível em: http://www.pns.icict.fiocruz.br/index.php?pag=proposicao

19. Barreto SM, Passos VM, Firmo JO, Guerra HL, Vidigal PG, Lima-Costa MF. Hypertension and clustering of cardiovascular risk factors in a community in Southeast Brazil: The Bambuí Health and Ageing Study. Arq Bras Cardiol. 2001;77(6):576-81. http://dx.doi.org/10.1590/S0066-782X2001001200008. PMid:11799432.

20. Consenso Brasileiro sobre Diabetes. Diagnóstico e classificação do diabetes mellitus e tratamento do diabetes mellitus tipo 2 [Internet]. São Paulo: Sociedade Brasileira de Diabetes; 2000 [citado em 2015 maio 8]. Disponível em: http://bvsms.saude.gov.br/bvs/publicacoes/consenso_bras_diabetes.pdf

21. Oliveira JEP, Montenegro RM Jr, Vencio S. Diretrizes da Sociedade Brasileira de Diabetes 2017-2018. São Paulo: Editora Clannad; 2017.

22. King P, Peacock I, Donnelly R. The UK Prospective Diabetes Study (UKPDS): Clinical and therapeutic implications for type 2 diabetes. Br J Clin Pharmacol. 1999;48(5):643-8. http://dx.doi.org/10.1046/j.13652125.1999.00092.x. PMid:10594464. 
23. Pinheiro R, Viacava F, Travassos C, Brito AS. Gênero, morbidade, acesso e utilização de serviços de saúde no Brasil. Ciênc Saúde Coletiva. 2002,7(4):687-707. http://dx.doi.org/10.1590/S1413-81232002000400007.

24. Joung IM, van der Meer JB, Mackenbach JP. Marital status and health care utilization. Int J Epidemiol. 1995;24(3):569-75. http://dx.doi.org/10.1093/ije/24.3.569. PMid:7672898.

25. Ribeiro FF Fo, Mariosa LS, Ferreira SRG, Zanella MT. Gordura visceral e síndrome metabólica: mais que uma simples associação. Arq Bras Endocrinol Metab. 2006;50(2):230-238.

26. Oliveira JEP, Vencio S. Diretrizes da Sociedade Brasileira de Diabetes: 2014-2015. São Paulo: AC Farmacêutica; 2015.

27. Gulve EA. Exercise and Glycemic Control in diabetes: benefits, challenges, and adjustments to pharmacotherapy. Physical Therapy. 2008;88(11):1297-1321.

28. Sigal RJ, Wasserman DH, Kenny GP, Castaneda C. Physical Activity/ Exercise and Type 2 Diabetes. Diabetes Care. 2004;27(10):2518-39. http://dx.doi.org/10.2337/diacare.27.10.2518. PMid:15451933.

29. Silva CA, Lima WC. Efeito benéfico do exercício físico no controle metabólico do Diabetes Mellitus Tipo 2 à curto prazo. Arq Bras Endocrinol Metab. 2002;46(5):550-56.

30. Mendes G, Rodrigues GBA, Nogueira JAD, Meiners MMMA, Lins TCL, Dullius J. Evidências sobre efeitos da atividade física no controle glicêmico: importância da adesão a programas de atenção em diabetes. Rev Bras Ativ Fis Saúde. 2013;18(4):412-23.

31. Ramalho ACR, Soares S. O papel do exercício no tratamento do diabetes melito Tipo 1. Arq Bras Endrocrinol Metab. 2008;52(2):260-67. 